\title{
Experimental investigation of the propagation properties of bloch surface waves on dielectric multilayer platform
}

\author{
Richa Dubey ${ }^{1 *} \mathbb{D}$, Elsie Barakat ${ }^{1}$, Markus Häyrinen², Matthieu Roussey², Seppo K. Honkanen², Markku Kuittinen² \\ and Hans Peter Herzig ${ }^{1}$
}

\begin{abstract}
Background: The periodic dielectric multilayers sustaining Bloch surface waves have been proposed as a platform for the sensing applications and the two dimensional integrated optics. In this paper, we present the experimental and theoretical investigation of propagation properties of Bloch surface waves, for example propagation length and refractive index of the surface mode, at the interface of a dielectric multilayer platform. We use thin layers ( N 25$)$ of titanium dioxide as an additional layer of high index material.

Methods: We exploit multi-heterodyne scanning near-field optical microscopy and total internal reflection configuration as a near-field and far-field characterization tools.

Results: The longest propagation length is achieved when the multilayer is designed to have the dispersion curve positioned close to the middle of the photonics band gap. We measure a Bloch surface wave mode of propagation length $3.24 \mathrm{~mm}$ and of an effective refractive index contrast 0.15 .
\end{abstract}

Conclusions: The experimental results are in conformity with theoretical results. This study paves a way to realize efficient and compact two dimensional components and systems.

Keywords: Bloch surface wave, Multilayer platform, Scanning near-field optical microscopy, Two-dimensional optics, Optical characterization, Optics at the surface

\section{Background}

Bloch Surface Waves (BSWs) have been known since the end of the 70's. After more than 30 years of their invention they have been recently considered as an alternative to surface plasmon polaritons (SPP), especially in sensing applications $[1,2]$. Similar to SPPs, BSWs are surface electromagnetic waves. BSW dispersion curves are located within the photonics bandgap (PBG) of a one dimensional photonic crystal. BSW show an exponential decay of the field inside the layered medium and in the air. Because of the tight confinement of the optical field to the multilayerair interface, a strong electromagnetic field enhancement arises. It should be noted that not only periodic, but also quasi-crystals and aperiodic dielectric multilayers can

\footnotetext{
* Correspondence: richa.dubey@epfl.ch

${ }^{1}$ Optics \& Photonics Technology Laboratory (OPT), École Polytechnique

Fédérale de Lausanne (EPFL), Neuchâtel CH-2002, Switzerland

Full list of author information is available at the end of the article
}

support BSWs. Examples include Bloch-like surface waves as proposed in the study of V. Koju et al. [3]. In further studies BSWs have been compared to SPPs and extensively exploited in sensing applications [4-12].

Moreover, the multilayer platform sustaining BSWs has been suggested for two dimensional (2D) integrated optics. It has been proven that BSWs can be guided, reflected, refracted, focused, diffracted, coupled and bent using 2D optical components such as ridge waveguides, lenses, prisms, gratings, waveguide couplers and curved waveguides on top of the multilayer platform [13-18]. These initial research works have paved the way for novel 2D integrated optics on the BSW platform.

Compared to SPPs, dielectric multilayers for BSWs have several advantages. First, due to low losses of dielectric materials, BSWs possess higher propagation lengths. Another advantage is the unlimited choice of materials constituting the multilayers. Of course the materials have 
to be transparent at the operating wavelength. Moreover, multilayers are wavelength scalable and may be designed to sustain BSWs at a broad range of wavelengths from nearUV to IR [5]. Since the position of the surface mode within the photonics band gap can be engineered by tailoring the thickness of the topmost layer of the multilayer, it is possible to tune the evanescent field content in the external medium. This ability to tune the localized field confinement is very appealing for sensing applications $[19,20]$.

The propagation length $L_{B S W}$ and effective refractive index $n_{\text {eff }}$ are the key parameters of BSWs, which determine the losses associated with the multilayer platform and the optical properties of the surface modes, respectively.

The decay of the amplitude of the surface mode along the propagation direction results from absorption in the material, surface scattering and leakage into the multilayer because of prism coupling. However, due to the low absorption of dielectric materials (constituent material) and to the low roughness of the multilayer surface, absorption and scattering do not have a significant contribution to the losses [21, 22]. The details about the constituent material can be found in the 'Method' section. Hence, the BSW is mainly decaying by light leakage into the prism. The decay coefficient of the surface mode due to leakage losses into the multilayer (neglecting the absorption and surface scattering losses) decreases exponentially as the number of periods of the multilayer increases [1].

The thin high refractive index layer on top of the BSW platform tends to increase the electromagnetic field confinement. The position of the surface mode inside the bandgap can be altered by tuning the thickness of this layer. This provides a better control of the optical properties of the surface mode, such as high propagation length or tight confinement to the surface. Furthermore, the thickness of this layer plays a key role in determining the BSW frequency (propagation vector), which can thus be tuned within the photonic band gap. The concept of using a thin layer enables the fabrication of customized 2D photonic elements at the surface of a standard multilayer. The main advantage is the ease of fabrication of $2 \mathrm{D}$ structures compared to $3 \mathrm{D}$ and the standard fabrication techniques used for the platform [14-18].

In this work, we mainly emphasize on studying the effect of thin layers on the BSW propagation properties by measuring the propagation lengths directly the in near-field.

\section{Methods}

\section{Constituent material and multilayer platform concept}

The multilayer platform consists of a dielectric stack, equivalent to a Bragg mirror, with periodically alternating high and low refractive index materials. The periodic structure consists of six periods of silicon dioxide $\left(\mathrm{SiO}_{2}\right)$ and silicon nitride $\left(\mathrm{SiN}_{\mathrm{x}}\right)$ having a refractive index of 1.45 and 1.79 around $\lambda=1550 \mathrm{~nm}$, respectively. Please note that the estimated imaginary part of the refractive indexes of $\mathrm{SiO}_{2}$ and $\mathrm{SiN}_{\mathrm{x}}$ are approximately $10^{-4}$ [23]. The values are selected to match with experimental investigations. The periodicity of the multilayer is terminated by a $50 \mathrm{~nm}$-thick terminating layer (top layer) of $\mathrm{SiN}_{\mathrm{x}}$. The thickness of the top layer determines the field profile of the BSW mode inside the multilayer [24]. The thicknesses of the $\mathrm{SiO}_{2}$ and $\mathrm{SiN}_{\mathrm{x}}$ layers are 472 and $283 \mathrm{~nm}$, respectively. The complete structure (Bragg mirror + top layer), called Bare Multilayer (BML), is deposited on a glass wafer as shown in Fig. 1(a). We exploit the BML as a platform for integrated optics which enables the wafer scale production and hence the low cost fabrication technology (see also Ref. [15]). The particular number of layers have been chosen to achieve optimum BSW excitation condition and low leakage losses. On one hand, decreasing the number of layers increases the coupling strength but on the other hand it increases the leakage losses and vice versa. We have optimized our design with six periods of $\mathrm{SiO}_{2}$ and $\mathrm{SiN}_{\mathrm{x}}$. At $\lambda=1558 \mathrm{~nm}$, the calculated reflectivity (matrix transfer method) of the Bragg mirror, inside the photonics bandgap for the incident angles greater than the critical angle (42 deg), is approximately 98.43\%. The BML exhibits a photonic band gap around telecom wavelengths and a BSW can be excited with TE and/or TM polarization. In the present work the multilayers are designed for TE polarization at the wavelength around $1550 \mathrm{~nm}$. As already mentioned, a topmost layer of a high refractive index material, which will be addressed as an additional layer hereafter, can be deposited on top of the BML. It can serve as an efficient device layer to shape micro/nano-photonic structures for further integrated optics applications. We use titanium dioxide $\left(\mathrm{TiO}_{2}\right)$ as an additional layer of high refractive index material. It has a refractive index of $2.23+\mathrm{i} 0$ at the wavelength of $1550 \mathrm{~nm}$ (measured by ellipsometry). The material is transparent in the visible and near-IR regions of light [22]. High refractive index provides strong in-plane confinement of the light and enables the development of compact and low loss complex shaped integrated 2D photonic devices. Here we study the effect of different layer thicknesses of $\mathrm{TiO}_{2}$ deposited on the BML on the BSW characteristics. The BML platform is fabricated using Plasma-Enhanced Chemical Vapor Deposition (PECVD, PlasmaLab $80+$ by Oxford Instruments), while Atomic Layer Deposition (ALD, TFS 200 by Beneq) is used for $\mathrm{TiO}_{2}$. This additional device layer has to be only a few tens of nanometers thick and ALD is an ideal method to deposit a homogenous coating all over the surface of the BML. This can be done at low temperature and with a very accurate control of the film thickness [22, 25, 26].

The dispersion diagram of the semi-infinite multilayer is plotted in Fig. 1(b). It shows the radiative (black) zones and non-radiative zones for propagation within the multilayer. The surface modes exist within the white zones that correspond to the photonic bandgap of the 


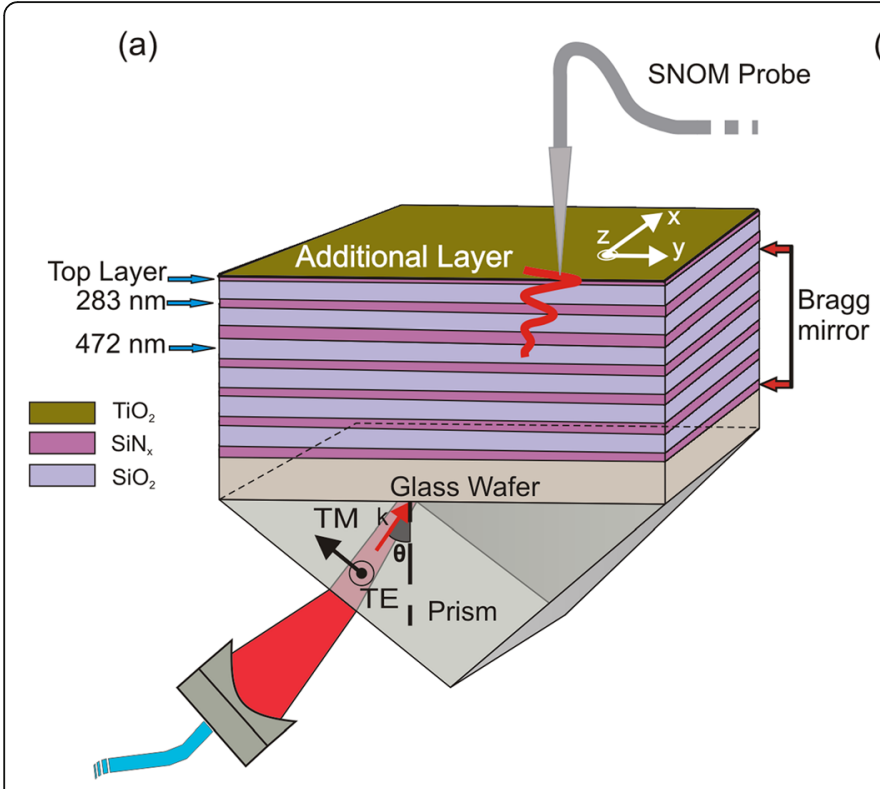

(b)

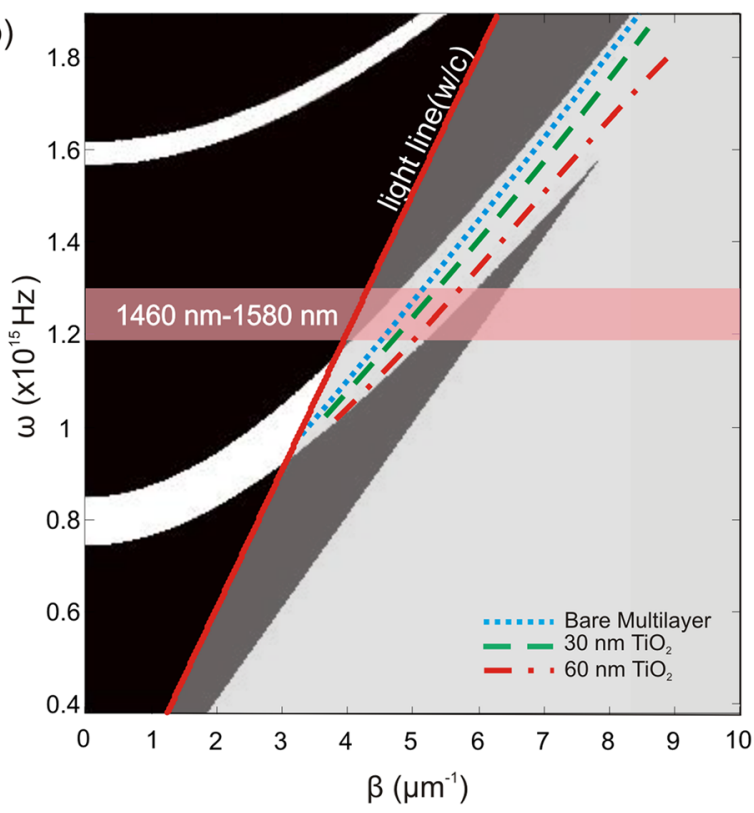

Focusing Lens

Fig. 1 Schematic of dielectric multilayer platform and corresponding dispersion band diagram. a Illustration of a dielectric multilayer based BSW platform deposited on a glass wafer. $\mathbf{b}$ Dispersion band diagram of the underlying multilayer with semi-infinite stacks for TE polarization

dispersion diagram. This means that the BSW cannot radiate either in air or in the multilayer. The dispersion band diagram of the underlying multilayer is calculated with the transfer matrix method [2]. The $\beta$ axis is the propagation constant $\beta=(2 \pi / \lambda) n \sin \theta$ of the incident light which is parallel to multilayers and the $\omega$ axis represents the angular frequency $\omega=2 \pi c_{0} / \lambda$, where $\lambda$ is the wavelength of incident light in vacuum, $n$ is the refractive index of the prism, $\theta$ is the incident angle and $c_{O}$ is the speed of light. The chosen parameters of the platform are such that the dispersion relation of the surface mode lies in the photonics band gap. The propagation properties of the surface mode on the platform can be altered by changing the thickness of the top layer or the additional layer. Their thicknesses define the location of the dispersion line in the band gap. It is revealed in the dispersion diagram, Fig. 1(b) that by increasing the thickness of the additional layers, the BSW dispersion line moves towards the edge of the band gap which means higher propagation constant at a fixed wavelength.

One can see from Fig. 1(b) that the dispersion relation of the BML and the $60 \mathrm{~nm}$ additional layer appear near the upper and lower edge of the bandgap, respectively, whereas the dispersion relation of the $30 \mathrm{~nm}$ layer of $\mathrm{TiO}_{2}$ lies in the middle of the bandgap. For the Bloch surface modes that occur near the center of the bandgap, the attenuation by the periodic multilayer stack is stronger and the mode is tightly confined to the surface. For the modes close to the edges, the attenuation is relatively weaker and the evanescent field penetrates much further into the multilayer $[19,24]$. Therefore, the PBG based platform can be designed based on the application behind. Strong field enhancement is needed for sensing and nonlinear applications while modes with longer propagation length are required for integrated optics.

Figure 2(a) shows the relation of the BSW dispersion line in the photonic bandgap for different thicknesses of top layer (red curve) and additional layer (blue curve). It should be noted that the calculations with the additional layer has been performed with fixed thickness of top layer $(50 \mathrm{~nm})$. One can see that by increasing either the additional or the top layer thickness, the corresponding propagation constant of the BSW mode increases. Since the additional layer has a higher refractive index, the propagation constant of BSW changes remarkably with the thickness. In Fig. 2(b), we plot in red the product of the period of multilayer and the absolute value of the imaginary part of the BSW wave vector $(\operatorname{Imag}(K))$ with respect to the effective index $(n \sin \theta)$. One can see that the values are higher in the middle of the bandgap which refers to the fact that attenuation by the PBG material is stronger. Consequently the evanescent field cannot penetrate deeper into the multilayer. The blue stars refer to the values of the (Imag $(K)$ ) for different thicknesses of the $\mathrm{TiO}_{2}$ layers. In green, we show the reflectivity spectrum of a platform with an additional layer of $60 \mathrm{~nm}$-thick $\mathrm{TiO}_{2}$ with respect to $n \sin \theta$. The dip 
(a)

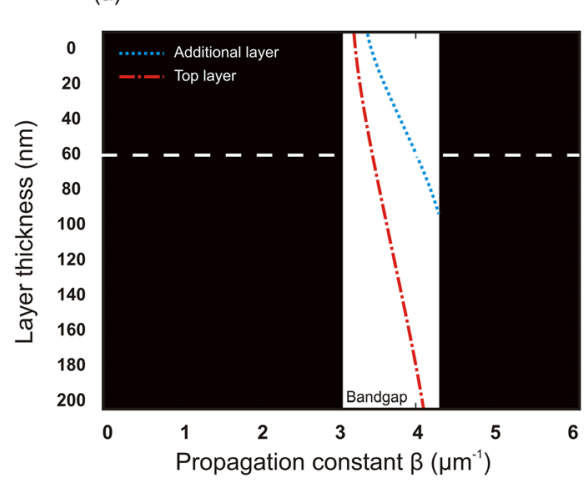

(b)

$\operatorname{abs}(\operatorname{Imag}(\mathrm{K} \wedge))$

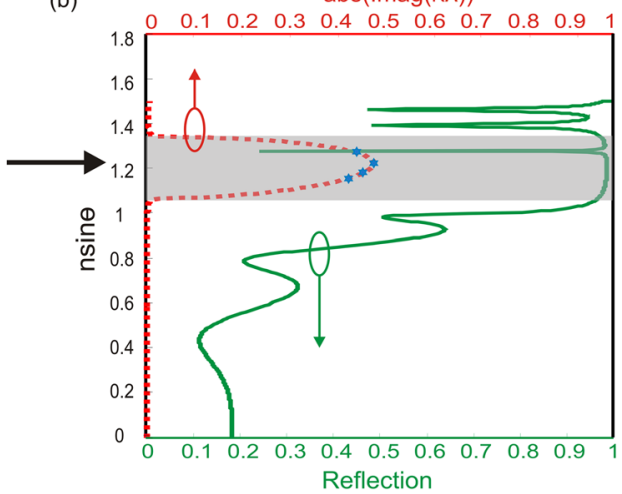

Fig. 2 Optical properties of BSW with respect to thicknesses of top/additional layer and effective index. a Evolution of the propagation constant of the BSW mode with respect to the top layer thickness (red) and the additional layer thickness (blue). b Reflection spectrum (green line) of $60 \mathrm{~nm}$ additional layer $\mathrm{TiO}_{2}$ and position in the photonic bandgap (greyed surface). The red spectrum shows the product of the period of multilayer and the absolute values of the imaginary part of the BSW wave vector which is perpendicular to the direction of BSW propagation. The values are lower at the edges of the bandgap. Blue stars correspond to the values of the imaginary part of the BSW wave vector for $60,30,15$ and 0 nm thicknesses of additional layer of $\mathrm{TiO}_{2}$ in the direction toward decreasing $n \sin \theta$ scale respectively

position in the reflectivity spectrum depends on the chosen parameters. Therefore, the additional layer and the top layer thicknesses can be tuned in order to modify the effective index of BSW depending on the targeted application.

\section{Experimental set up for Bloch surface wave excitation and characterization}

In the experiments done in this paper, a total internal reflection (TIR) configuration is used for BSW coupling. The TIR configuration is needed for BSW excitation since its wave vector lies beyond the light line. Thus, it is not possible to couple the surface wave directly in air. A schematic of the total internal reflection configuration is shown in Fig. 1(a). A BK7-glass prism is used for the coupling $(n=1.501)$. The laser beam incidents at the prism at an angle $\theta$ which is higher than critical angle. At a particular angle and wavelength, the incident light is coupled to BSW which can be seen as a reflection dip of the angular reflectance plot in the far-field (FF). The incident angle $\vartheta$ is chosen so that the parallel component of the wave vector of the incident light matches the following condition, $\beta=K \sin \theta$ where, $K=2 \pi n / \lambda$ is the wave vector of the incident beam, $n$ is the refractive index of the prism, $\lambda$ is the wavelength of the incident light in vacuum and $\beta$ is the propagation constant of the surface mode. The laser source is an external cavity diode laser (Agilent, Model 81682A) tunable for wavelengths ranging from 1460 to $1580 \mathrm{~nm}$. We use a focused Gaussian beam to couple light onto the surface of the multilayer. An incident beam illuminates the prism at an angle $\theta=50.15$ deg., for bare multilayer (BML), at the corresponding wavelength $\lambda=1558 \mathrm{~nm}$. In our case, the horizontal spot diameter in the coupling zone is around $30 \mu \mathrm{m}$. The surface wave propagates in the $x-y$ direction as shown in Fig. 1(a).

Since a BSW is an evanescent mode, the near-field (NF) can be collected by a scanning near field optical microscopy (SNOM) with a subwavelength aperture probe (in collection mode) appended to a single mode fiber. The sample illumination system is placed in the object arm of a multi-heterodyne SNOM (MH-SNOM). We exploit the MH-SNOM to observe and measure the surface wave propagation at the top of the sample [27]. The MH-SNOM is an upgraded version of a classical heterodyne SNOM. Unlike in the heterodyne interferometer, object and reference beams are further divided into two orthogonally polarized object and reference beams, respectively with a beam splitter having a 50/50 splitting ratio. First, the laser beam is sent to a polarization controller before passing through a polarization beam splitter. The purpose of the polarization controller is to adjust the ratio of object and reference powers at the output of the polarization beam splitter. Each of the four channels (object and reference for both polarizations) is then modulated with different frequencies. These frequencies are generated by two separate acousto-optic modulators. This is done to obtain simultaneously information on the object amplitude and phase in terms of beat frequencies. The two object beams are recombined into a polarization-maintaining fiber, this fiber is rotated to generate TE and TM illumination beams. The reference beams are recombined into a single mode fiber. Their orthogonality and linearity are well preserved up to the detector. The object beam illuminates the sample. After the illumination system, two object and two reference beams are recombined into a single mode fiber with a beam splitter having a 99/1 splitting ratio. The superimposition of 
four different frequencies (two object beams, two reference beams) on the detector generates six different beat frequencies. These different beat frequencies can be detected with the help of lock-in amplifiers. Each of them is locked to one corresponding beat frequency. More details about MH-SNOM can be found in [27, 28].

A schematic of the far-field experimental setup is shown in Fig. 3. A collimated and TE-polarized beam is used to illuminate the sample through the input slant facet of a glass prism. The angular divergence of the collimated incident beam is measured (from the spot size) to be $\phi=0.0024 \mathrm{rad}$. The sample is contacted with the prism flat facet using a proper index matching liquid. On the other side of the prism, the reflected light is collected by a Germanium photodiode detector. The sample and the detector are placed on a rotational stage, which is driven by a stepper motor controller. The rotational stage is being used to rotate the sample with respect to the incident beam. The angle ' $\theta$ ' is measured between the normal to the multilayer planar interface and the direction of the incident beam at the prism. The angular reflectance profiles at a fixed wavelength are obtained by scanning a range of angles $(\theta)$.

\section{Results and discussion}

\section{Propagation length analysis}

As a BSW propagates along the surface, it looses energy to the multilayer system. Thus, the propagation length is an important property of a BSW. It is the distance where the amplitude of the propagating field drops down exponentially $\left(\exp \left(-y / L_{B S W}\right)\right)$ to $1 / e$ of its maximum value. As described in the introduction, the main source of mode loss is the leakage into the multilayer because of the presence of the prism. The multilayer design has been optimized for low losses by appropriately choosing the thicknesses and number of periods. The propagation

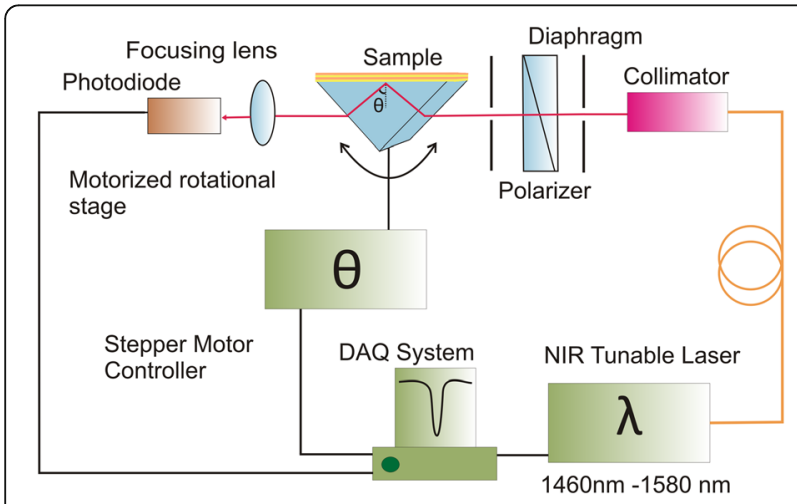

Fig. 3 Schematic of the far-field experimental set up. Schematic of the far-field experimental set up in total internal reflection configuration used for far-field characterization of BSWs lengths for the BML and BML with different thicknesses of the additional layer $\left(\mathrm{TiO}_{2}\right)$ have been estimated by measuring near-fields.

A near-field image of BSW propagation at wavelength $1558 \mathrm{~nm}$ is shown in Fig. 4(a). The image represents the field amplitude distribution of BSW propagation, where the evanescent field is collected by a MH-SNOM probe. The amplitude distribution map is analysed to deduce the BSW beam divergence and propagation length in $\mathrm{x}$ and y direction, respectively, as shown in Fig. 4(a). $L_{B S W}$ is obtained by exponentially fitting the decrease of the field amplitude of the surface wave along the propagation direction where the region with maximum field amplitude represents the coupling zone, as demonstrated in Fig. 4(b). We have calculated an error margin using a standard absolute error analysis method. Mainly, the divergence of the BSW and the surface quality influence the propagation length. Hence, error calculations include the divergence of the BSW, surface nonuniformity and also the reproducibility of the results. To determine the divergence of the propagating BSW, the full width half maximum (FWHM) of the measured beam profile (in $x$ direction, Fig. 4(a)) has been calculated at equidistant positions along the direction of propagation for one millimeter. For BML, the measured value of the divergence angle in the near-field is approximately $0.27 \mathrm{deg}$. Note that the perfect conformal coating provided by ALD insures a homogeneity of the layer thickness all over the sample even in tiny apertures. A small reduction of the surface roughness can also be observed for thicker layer [22]. For $30 \mathrm{~nm}$ thickness of $\mathrm{TiO}_{2}$, we achieved a propagation length of around $L_{B S W}^{N F}=3.24 \pm$ $0.13 \mathrm{~mm}$ in the near-field, which is nearly 40 times longer than the recently obtained "Long-Range SPPs" studied by J. Lin et al. [29] and 5 times longer than the one studied by L. Yu et al. [15].

The propagation length of the $\mathrm{BSW}$, without an additional layer (BML) and for other thicknesses of $\mathrm{TiO}_{2}$ have also been measured. Different thicknesses of the $\mathrm{TiO}_{2}$ layer have been deposited on different BML samples with the same multilayer platform design. The measured propagation lengths for the BML, with $15 \mathrm{~nm}$ of $\mathrm{TiO}_{2}, 30 \mathrm{~nm}$ of $\mathrm{TiO}_{2}$ and $60 \mathrm{~nm}$ of $\mathrm{TiO}_{2}$ are $1.35 \pm 0.09$, $2.77 \pm 0.15,3.24 \pm 0.13$ and $2.53 \pm 0.14 \mathrm{~mm}$, respectively. The corresponding propagation losses in $\mathrm{dB} / \mathrm{mm}$ $\left(8.68 / L_{B S W}\right)$ are $6.42,3.13,2.68$ and $3.43 \mathrm{~dB} / \mathrm{mm}$ for the BML, with $15 \mathrm{~nm}$ of $\mathrm{TiO}_{2}, 30 \mathrm{~nm}$ of $\mathrm{TiO}_{2}$ and $60 \mathrm{~nm}$ of $\mathrm{TiO}_{2}$, respectively. The propagation lengths with error margins have been plotted against different thicknesses of $\mathrm{TiO}_{2}$ in Fig. 4(c). The rigorous calculations to deduce the $L_{B S W}$ have been performed by $\mathrm{M}$. Menotti et al. [30]. It has been explained that $L_{B S W}$ is directly related to the Imag $(K)$ and the number of periods of the multilayer. In the present design of the 


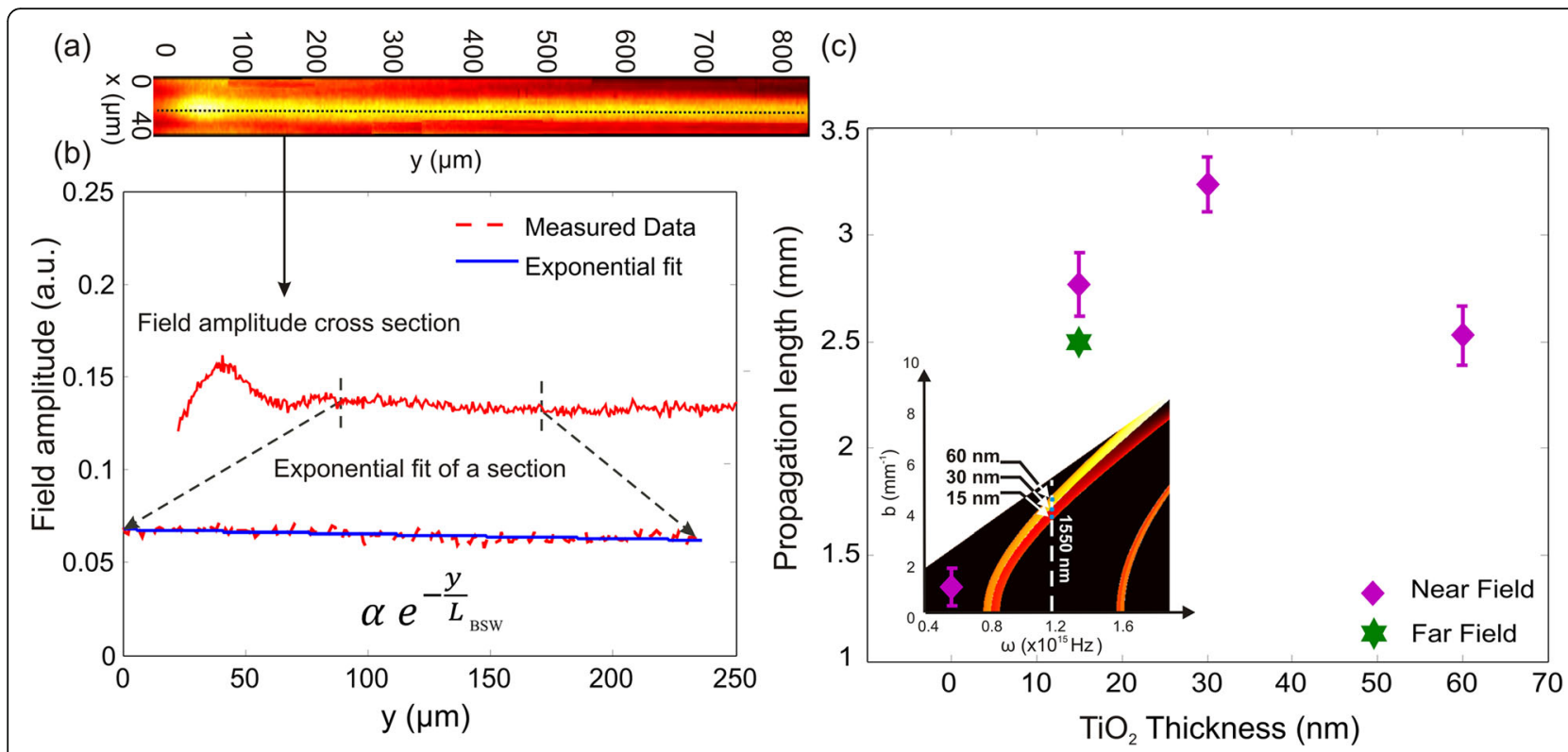

Fig. 4 Propagation length measurement for different thicknesses of additional layer with the aid of MH-SNOM. a MH-SNOM image demonstrating BSW propagation in the near-field. $\mathbf{b}$. Exponential fit of the field amplitude along the direction of propagation to measure the propagation length. c Near-field measurement of propagation lengths with the error margin for $\mathrm{BML}\left(0 \mathrm{~nm} \mathrm{TiO}{ }_{2}\right), 15 \mathrm{~nm}$ of $\mathrm{TiO}_{2}, 30 \mathrm{~nm}$ of $\mathrm{TiO}_{2}$ and $60 \mathrm{~nm}$ of $\mathrm{TiO}_{2}$ thicknesses

multilayers the value of Imag $(K)$, which controls the damping of the electric field in the multilayer, is maximum at the middle of the PBG (Referring to Fig. 2(b)). This implies, the evanescent field cannot penetrate deeper into the multilayer close to the middle of the band gap. Therefore $L_{B S W}$ is longest in the middle of the PBG, since the leakage is the dominating propagation loss mechanism. For example, referring to the propagation length plot and the dispersion band diagram [Figs. 1(b) and 4(c) inset], the propagation length for a $30 \mathrm{~nm}$-thick $\mathrm{TiO}_{2}$ layer is the longest compared to other thicknesses and the dispersion line lies at the middle of the bandgap.

The propagation length of prism-coupled waveguide modes can also be deduced from the bandwidth of reflectance anomalies, as described by Ulrich in [31]. The reflectance spectrum as a function of the incident angle $\theta$ is measured using a far-field set up as explained under the methods section (see Fig. 3). Notwithstanding, measured reflectance dips broaden significantly due to the angular divergence of the illuminating beam. In fact, for the given coupling system, the reflectance anomaly is a result of the convolution of the multilayer waveguide transfer function and the angular spectrum of the incident beam. In the far-field characterization method, we use a Gaussian beam whose measured angular divergence is about $\phi=0.0024 \mathrm{rad}$. The corresponding beam waist is around $w_{0}=\lambda / \phi \pi=206 \mu \mathrm{m}$. We removed the effect of the finite size of the beam width (by deconvolution) and estimated the FWHM of the reflectance curve as around $\sigma=2 \mathrm{x} 10^{-4}$, which approximates the BSW losses for a plane-wave excitation. As an example, we show here the propagation length of the BSW mode for the additional layer of $15 \mathrm{~nm} \mathrm{TiO}{ }_{2}$. The estimated value in the farfield for prism-film coupling systems is following [31]:

$$
L_{B S W}^{F F}=\lambda / \pi \sigma=2.5 \text { millimeters, }
$$

which is very close to the near-field propagation length of $15 \mathrm{~nm}$ of $\mathrm{TiO}_{2}(\sim 2.7 \mathrm{~mm})$.

\section{Effective refractive index analysis}

An effective refractive index $n_{\text {eff }}$ is introduced by the propagation constant of surface waves. When the multilayer is coated with ultra-thin films, the effective refractive index shifts to a higher value creating a contrast between the BML and the coated multilayer. The refractive index contrast $(\Delta n)$ mainly affects the capability to manipulate the BSW. The refractive index contrast, produced by optical elements on the top of the multilayer, plays a key role in determining the optical properties of the surface photonic devices and hence their efficiency in the manipulation of the BSW. High $\Delta n$ provides strong lateral confinement of the light inside the waveguide structures and enables the development of compact and low loss integrated 2D photonic devices. Here, we measure $\Delta n$ of the propagating BSW for different thicknesses of $\mathrm{TiO}_{2}$ in near-field and far-field. To perform this measurement in the near-field, a high resolution map of the phase is acquired using the $\mathrm{MH}$ SNOM. From a phase cross sectional profile, shown in Fig. 5, one can calculate the effective refractive index 


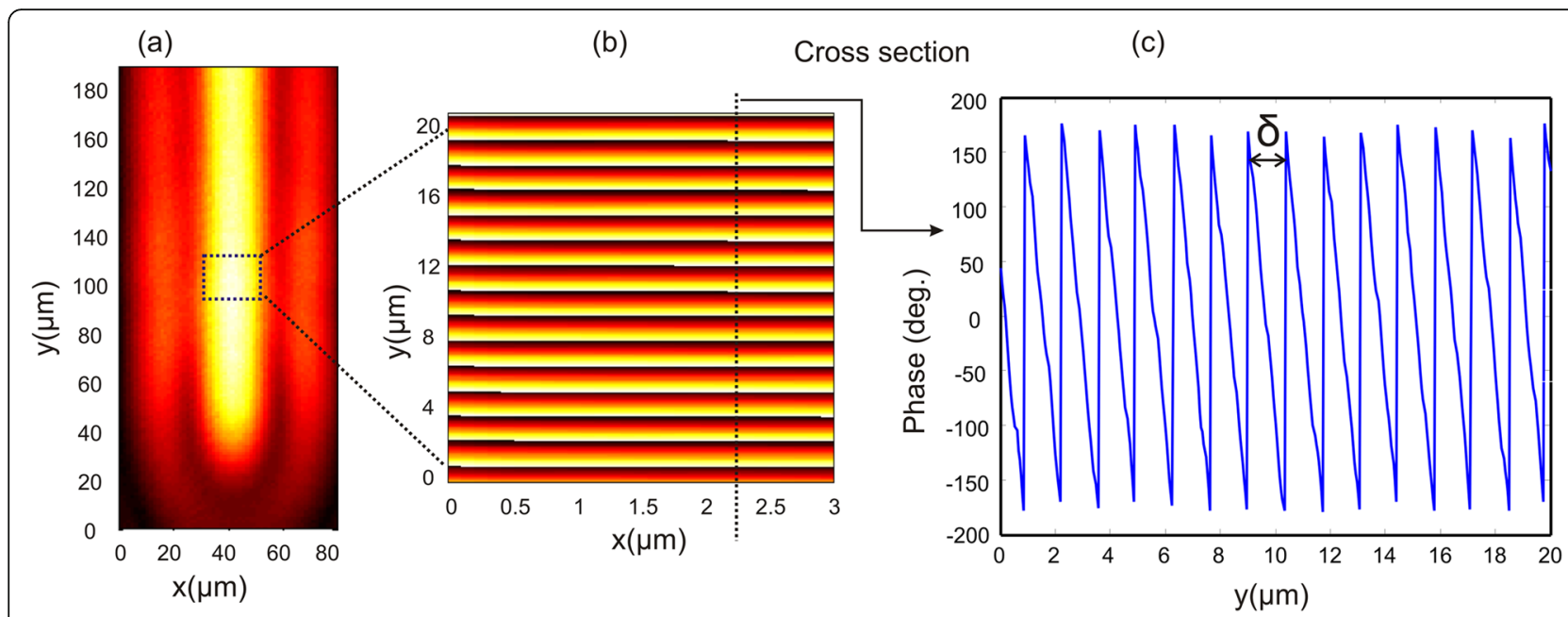

Fig. 5 Measurement of BSW phase with the aid of MH-SNOM. a Near-field MH-SNOM image of BSW propagation. $\mathbf{b}$ High resolution (400 points in $20 \mu \mathrm{m}$ scan length) map of phase along the direction of BSW propagation. c Cross section of BSW phase along the propagation direction

contrast using simple transformations in the Fourier domain:

$$
\begin{aligned}
& \lambda \text { eff }=\frac{1}{f_{\mathrm{s}}}=\delta, \\
& n_{\text {eff }}=\frac{\lambda}{\lambda_{\mathrm{eff}}}=\frac{\lambda}{\delta}, \\
& \Delta n=n_{\mathrm{TiO} 2}-n_{\mathrm{P}},
\end{aligned}
$$

where $f_{s}$ is the spatial frequency, $\delta$ is the period of the phase diagram, $n_{\mathrm{TiO} 2}$ and $n_{p}$ are the effective refractive indices with and without the $\mathrm{TiO}_{2}$ layer (BML platform), respectively. In the near-field, a $\Delta n$ of 0.15 has been obtained for a $60 \mathrm{~nm}$ thick $\mathrm{TiO}_{2}$ layer which is significantly higher than the $100 \mathrm{~nm}$ thick layer of photoresist [15].

In addition, $n_{\text {eff }}$ has also been measured in the farfield. The far-field set up has been discussed under the methods section (see Fig. 3). Figure 6(a) shows a typical reflectance spectrum as a function of the prism incident angle $\theta$. The red curve corresponds to the bare multilayer where there is no additional layer. The yellow and green curves correspond to the $15 \mathrm{~nm}$ and $60 \mathrm{~nm}$-thick additional $\mathrm{TiO}_{2}$ layers, respectively. The result plots only a small angular range in the vicinity of the reflectivity dip. The dips correspond to the Bloch surface modes propagating at the multilayer-air interface. By adding the additional layer, the surface mode is moved to the middle and to the lower edge of the bandgap thus to larger parallel wave vectors expressed with a high incident angle. The effective refractive index, $n_{\text {eff }}=n \sin \theta$, can be deduced from the dip of the reflectance for the different $\mathrm{TiO}_{2}$ layer thicknesses. A maximum effective refractive index contrast $\Delta n=0.16 \pm 0.01$ is obtained for $60 \mathrm{~nm}$ thickness of $\mathrm{TiO}_{2}$. The error margin has been introduced from the calibration of the incident angle $\theta$. The resolution of the stepper motor is $0.0025 \mathrm{deg} / \mathrm{step}$. The
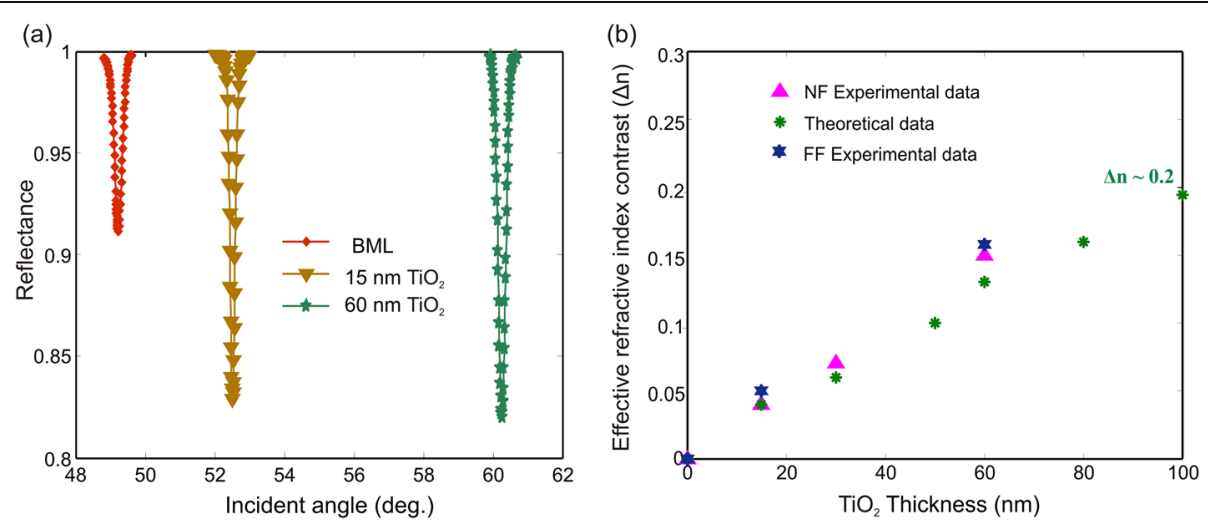

Fig. 6 Reflectance spectrum and Effective refractive index contrast with respect to different thicknesses of additional layer. a Reflection as a function of the incident angle for different $\mathrm{TiO}_{2}$ layer thicknesses, measured in the far-field. $\mathbf{b}$ The effective refractive index contrast of a $\mathrm{BSW}$ for different thicknesses of $\mathrm{TiO}_{2}$ layer (from 15 to $100 \mathrm{~nm}$ ) in near-field (NF), theory and far-field (FF) 
calculated error in $n_{\text {eff }}$ is, $\Delta n_{\text {eff }}=n \Delta \theta \cos \theta$. We plotted the theoretical (matrix transfer method), the near-field and the far-field measurement results of $\Delta n$ as a function of the $\mathrm{TiO}_{2}$ thicknesses in Fig. 6(b). The experimental and theoretical results show a good agreement with each other. The simulation results show that a $\Delta n$ close to 0.2 can be achieved for $100 \mathrm{~nm}$ of $\mathrm{TiO}_{2}$ thickness.

\section{Conclusion}

The propagation length of Bloch surface waves at the interface of a multilayer platform has been studied experimentally. The objective of the study is to have more insight to design BSW based integrated optical systems. Different additional layer thicknesses $(\sim \lambda / 25)$ of a high refractive index material, in our case $\mathrm{TiO}_{2}$, have been investigated. Another important property of BSW, for example the effective refractive index, has been presented as well. These properties are measured in the near-field and far-field. The near-field characterization has been done with the aid of a multi-heterodyne scanning near field optical microscope and the far- field by measuring the incident angle at resonance. We have measured the influence of the thickness and refractive index of the additional (device) layer on the propagation properties of BSWs. A longer propagation length $(3.24 \mathrm{~mm})$ is achieved near to the middle of the photonic band gap where theory suggests the stronger attenuation. However, this is not the limit, higher values (in the order of centimeters) might be achieved using grating couplers. Most importantly, by tailoring the thickness of the additional layer a surface mode can be shaped according to the application, for example integrated optics or sensing. High refractive index is preferred for low loss and compact photonic devices. We achieve a $\Delta n$ close to 0.15 for $60 \mathrm{~nm}$ of $\mathrm{TiO}_{2}$. The theoretical results demonstrate that we can reach a $\Delta n$ close to 0.2 for $100 \mathrm{~nm}$ thickness of $\mathrm{TiO}_{2}$. The experimental results show a good agreement with the theoretical results. In addition to the valuable increase of the propagation length of the BSW, we have also demonstrated that an additional layer allows fine tuning of the BSW frequency, which can thus be positioned at any place within the photonic band gap. These novel features make the BSW based multilayer platform more efficient for further integrated photonics applications. Considering the strong interest in BSWs, we believe that the presented results can provide a better perception to design application oriented devices.

\footnotetext{
Abbreviations

BSW: Bloch surface wave; FF: Far- field; $L_{B S W}$ : Propagation length; MH-SNOM: Multi-heterodyne scanning near-field optical microscopy; $n_{\text {eff: }}$ Effective refractive index; NF: Near-field; TE: Transverse electric; TIR: Total internal reflection; TM: Transverse magnetic; $\Delta n$ : Effective refractive index contrast
}

Funding

We acknowledge funding from Swiss National Science Foundation (SNSF) (200020_135455) and Finnish Funding Agency for Innovation under project Tekes FiDiPro NP-NANO (No 40315/13).

\section{Availability of data and materials}

The datasets supporting the conclusions of this article are included within the article.

\section{Authors' contributions}

RD performed near-field experiments, far-field experiments, data analysis and wrote the manuscript. EB worked on the theoretical part. MH performed the atomic layer deposition, MR and MK supervised the work at university of Eastern Finland. HPH proposed the fundamental concept. The final manuscript is the result of common contributions and discussions. All the authors read and approved the final manuscript.

\section{Competing interests}

The authors declare that they have no competing interests.

\section{Author details}

${ }^{1}$ Optics \& Photonics Technology Laboratory (OPT), École Polytechnique Fédérale de Lausanne (EPFL), Neuchâtel CH-2002, Switzerland. ${ }^{2}$ Institute of Photonics, University of Eastern Finland, Joensuu 80101, Finland.

Received: 14 October 2016 Accepted: 14 December 2016

Published online: 25 January 2017

\section{References}

1. Yeh, P., Yariv, A., Cho, A.Y.: Optical surface waves in periodic layered media. Appl Phys Lett 32(2), 104-105 (1978)

2. Yeh, P., Yariv, A., Hong, C.-S.: Electromagnetic propagation in periodic stratified media. I. General theory. J Opt Soc Am 67(4), 423-438 (1977)

3. Koju, V., Robertson, W.M.: Excitation of Bloch-like surface waves in quasi-crystals and aperiodic dielectric multilayers. Opt Lett 41(13), 2915-2918 (2016)

4. Shinn, M., Robertson, W.M.: Surface plasmon-like sensor based on surface electromagnetic waves in a photonic band-gap material. Sens Actuators B Chem 105(2), 360-364 (2005)

5. Sreekanth, K.V., Zeng, S., Shang, J., Yong, K.-T., Yu, T.: Excitation of surface electromagnetic waves in a graphene-based Bragg grating. Sci Rep 2, 737 (2012)

6. Pirotta, S., Xu, X.G., Delfan, A., Mysore, S., Maiti, S., Dacarro, G., Patrini, M., Galli, M., Guizzetti, G., Bajoni, D.: Surface-enhanced Raman scattering in purely dielectric structures via Bloch surface waves. J Phys Chem C 117(13), 6821-6825 (2013)

7. Delfan, A., Liscidini, M., Sipe, J.E.: "Surface enhanced Raman scattering in the presence of multilayer dielectric structures". J Opt Soc Am B 29(8), 1863-1874 (2012)

8. Giorgis, F., Descrovi, E., Summonte, C., Dominici, L., Michelotti, F.: Experimental determination of the sensitivity of Bloch surface waves based sensors. Opt Express 18(8), 8087-8093 (2010)

9. Sinibaldi, A., Danz, N., Descrovi, E., Munzert, P., Schulz, U., Sonntag, F., Dominici, L., Michelotti, F.: Direct comparison of the performance of Bloch surface wave and surface plasmon polariton sensors. Sens Actuators B Chem 174, 292-298 (2012)

10. Danz, N., Sinibaldi, A., Michelotti, F., Descrovi, E., Munzert, P., Schulz, U., Sonntag, F.: Improving the sensitivity of optical biosensors by means of Bloch surface waves. Biomed Tech 57(1), 584-587 (2012)

11. Liscidini, M., Sipe, J.E.: Analysis of Bloch-surface-wave assisted diffractionbased biosensors. J Opt Soc Am B 26(2), 279-289 (2009)

12. Paeder, V., Musi, V., Hvozdara, L., Herminjard, S., Herzig, H.P.: Detection of protein aggregation with a Bloch surface wave based sensor. Sens Actuators B Chem 157(1), 260-264 (2011)

13. Descrovi, E., Sfez, T., Quaglio, M., Brunazzo, D., Dominici, L., Michelotti, F., Herzig, H.P., Martin, O.J.F., Giorgis, F.: Guided bloch surface waves on ultrathin polymeric ridges. Nano Lett 10(6), 2087-2091 (2010)

14. Sfez, T., Descrovi, E., Yu, L., Quaglio, M., Dominici, L., Nakagawa, W., Michelotti, F., Giorgis, F., Herzig, H.P.: "Two-dimensional optics on silicon nitride multilayer: Refraction of Bloch surface waves". Appl Phys Lett 96(15), 151101 (2010) 
15. Yu, L., Barakat, E., Sfez, T., Hvozdara, L., Di Francesco, J., Peter Herzig, H.: "Manipulating Bloch surface waves in 2D: a platform concept-based flat lens". Light Sci Appl 3(1), 124 (2014)

16. Yu, L., Barakat, E., Di Francesco, J., Herziq, H.P.: Two-dimensional polymer grating and prism on Bloch surface waves platform. Opt Express 23(25), 31640-31647 (2015)

17. Yu, L., Barakat, E., Nakagawa, W., Herzig, H.P.: Investigation of ultra-thin waveguide arrays on a Bloch surface wave platform. J Opt Soc Am B 31(12), 2996-3000 (2014)

18. Wu, X, Barakat, E, Yu, L, Sun, L, Wang, J, Tan, Q and Herzig, HP: "Phase-sensitive near field Investigation of Bloch surface wave propagation in curved waveguides". J Eur Opt Soc Rapid Publ. 9, 14049-1-14049-8 (2014)

19. Robertson, W.M.: Experimental measurement of the effect of termination on surface electromagnetic waves in one-dimensional photonic bandgap arrays. J Light Technol 17(11), 2013-2017 (1999)

20. Konopsky, V.N., Alieva, E.V.: Photonic crystal surface waves for optical biosensors. Anal Chem 79(12), 4729-4735 (2007)

21. Bontempi, E., Depero, L.E., Sangaletti, L., Giorgis, F., Pirri, C.F.: Growth process analysis of a-Si1 - XNx:H Films probed by X-Ray Reflectivity. Mater Chem Phys 66(2-3), 172-176 (2000)

22. Hayrinen, M., Roussey, M., Gandhi, V., Stenberg, P., Saynatjoki, A., Karvonen, L, Kuittinen, M., Honkanen, S.: Low-loss titanium dioxide strip waveguides fabricated by atomic layer deposition. J Light Technol 32(2), 208-212 (2014)

23. Paeder, V: Bloch surface wave biosensing: study of optical elements for the early detection of protein aggregation. (PhD Dissertation, École Polytechnique Fédérale de Lausanne, 2011)

24. Gaspar-Armenta, J.: Surface waves in finite one-dimensional photonic crystals: mode coupling. Opt Commun 216(4-6), 379-384 (2003)

25. Puurunen, R.L.: "A short history of atomic layer deposition: Tuomo Suntola's atomic layer epitaxy: a short history of atomic layer". Chem Vap Depos 20(10-11-12), 332-344 (2014)

26. Dubey, R., Vosoughi Lahijani, B., Barakat, E., Häyrinen, M., Roussey, M., Kuittinen, M., Herzig, H.P.: Near-field characterization of a Bloch-surfacewave-based 2D disk resonator. Opt Lett 41 (21), 4867-4870 (2016)

27. Sfez, T., Descrovi, E., Yu, L., Brunazzo, D., Quaglio, M., Dominici, L., Nakagawa, W. Michelotti, F., Giorgis, F., Martin, O.J.: Bloch surface waves in ultrathin waveguides: near-field investigation of mode polarization and propagation. J Opt Soc Am B 27(8), 1617-1625 (2010)

28. Sfez, T: Investigation of surface electromagnetic waves with multiheterodyne scanning near-field optical microscopy. (PhD Dissertation, École Polytechnique Fédérale de Lausanne, 2010)

29. Lin, J., Dellinger, J., Genevet, P., Cluzel, B., De Fornel, F., Capasso, F.: "CosineGauss plasmon beam: a localized long-range nondiffracting surface wave". Phys Rev Lett 109(9), 93904 (2012)

30. Menotti, M., Liscidini, M.: Optical resonators based on Bloch surface waves. J Opt Soc Am B 32(3), 431-438 (2015)

31. Ulrich, R.: Theory of the prism-film coupler by plane-wave analysis. J Opt Soc Am 60(10), 1337-1350 (1970)

\section{Submit your manuscript to a SpringerOpen ${ }^{\circ}$ journal and benefit from:}

- Convenient online submission

- Rigorous peer review

Immediate publication on acceptance

- Open access: articles freely available online

- High visibility within the field

- Retaining the copyright to your article 